\title{
A Novel Charging and Discharging Algorithm of Plug-in Hybrid Electric Vehicles Considering Vehicle-to-Grid and Photovoltaic Generation
}

\author{
Ahmed Aljanad ${ }^{1}$, Azah Mohamed ${ }^{1}$, Tamer Khatib ${ }^{2, *}$, Afida Ayob ${ }^{1}$ and Hussain Shareef ${ }^{3}(\mathbb{D}$ \\ 1 Center for Integrated Systems Engineering and Advanced Technologies, University Kebangsaan Malaysia, \\ Bangi 43600, Selangor, Malaysia; amj442@gmail.com (A.A.); azah_mohamed@ukm.edu.my (A.M.); \\ afida@eng.ukm.my (A.A.) \\ 2 Department of Energy Engineering and Environment, An-Najah National University, Nablus 97300, Palestine \\ 3 Department of Electrical Engineering, United Arab Emirates University, Al-Ain 15551, UAE; \\ shareef@uaeu.ac.ae \\ * Correspondence: t.khatib@najah.edu; Tel.: +970-599317172
}

Received: 15 July 2019; Accepted: 17 September 2019; Published: 27 September 2019

\begin{abstract}
Considering, the high penetration of plug-in electric vehicles (PHEVs), the charging and discharging of PHEVs may lead to technical problems on electricity distribution networks. Therefore, the management of PHEV charging and discharging needs to be addressed to coordinate the time of PHEVs so as to be charged or discharged. This paper presents a management control method called the charging and discharging control algorithm (CDCA) to determine when and which of the PHEVs can be activated to consume power from the grid or supply power back to grid through the vehicle-to-grid technology. The proposed control algorithm considers fast charging scenario and photovoltaic generation during peak load to mitigate the impact of the vehicles. One of the important parameters considered in the CDCA is the PHEV battery state of charge (SOC). To predict the PHEV battery SOC, a particle swarm optimization-based artificial neural network is developed. Results show that the proposed CDCA gives better performance as compared to the uncoordinated charging method of vehicles in terms of maintaining the bus voltage profile during fast charging.
\end{abstract}

Keywords: charging and discharging control algorithm; state of charge; plug-in hybrid electric vehicle; particle swarm optimization; artificial neural network

\section{Introduction}

The emergence of plug-in electric vehicles (PHEVs) raises concerns regarding its impact on the power distribution systems, especially in the case of large-scale deployment of PHEVs [1-3]. As a fact, generation adequacy, load diagram modification and electricity grid robustness should be studied in accordance with the existing power grid when large amounts of PHEVs are deployed [4,5]. Many studies have been conducted regarding the impact of PHEVs on the grid during normal charging and uncoordinated charging when they are connected randomly in a distribution system [6]. In general, these studies consider voltage deviation, power losses and line loading as the most important technical issues.

With the integration of renewable energy sources in a smart grid, the energy supply which fluctuates and has unpredictable characteristics, will not be able to meet the needs of a dynamic and unpredictable load demand. To overcome this problem, vehicle-to-grid (V2G) systems are considered as a promising solution. In V2G systems, PHEVs can be used as distributed energy storage devices in a smart grid. The PHEV's battery can be either part of the load or a generator because most of the time the vehicles are idle at homes, in parking lots, or garages. Moreover, the time during which PHEVs are 
in parking lots is typically longer than the time required for charging them. Thus, it is possible for PHEVs to provide an opportunity to implement V2G services. The PHEV battery capacity is usually limited, but it is suitable for short-time ancillary services given its small response time as well as lower capital costs [7]. Considering that, the V2G operation will provide extra benefits to the vehicle owners and thus, there is a need to develop an effective charging and discharging algorithm for multiple PHEV batteries to optimize the energy consumption profile and to provide incentives for PHEV owners to participate in the discharging process.

Several works have been carried out using optimization and intelligent techniques to determine charging schedules for PHEV batteries. In a study by Han et al. [8], the charging sequence control problem for an electric vehicle was studied to maximize its revenue in each charging period. The dynamic programming technique is applied to determine the optimal charging sequence that would maximize the profit while satisfying the state-of-charge level required at the end of the charging period. Coordinated PHEV charging problem for minimizing power loss and voltage deviations was also studied by developing an algorithm for coordinated charging of PHEV batteries [9]. In another study by Deilami et al. [10], a real-time smart load management algorithm was developed for coordinating the charging of multiple PHEVs in a smart grid system to minimize power loss and improve voltage profile. An autonomous distributed V2G control scheme that includes charging request, battery condition and contribution to the smart grid was also studied [11]. In [12] which is conducted by Singh et al., the fuzzy logic control technique was applied to develop V2G controllers for controlling the power flow between a node and the charging station to meet peak power demand. In [13], a game theoretical approach was applied to a design in which the players were electric vehicles and their strategies were the control decisions on energy charging or discharging.

In the recent literature, heuristic optimization techniques have been applied for optimal charging and discharging of PHEVs. Meanwhile, the problem of controlling vehicles state of charge (SOC) in real time is addressed by utilizing evolutionary optimization and a self-adaptive strategy in [14]. In the paper proposed by Wang and Liang [15], a new charging scheme called water falling is introduced to achieve optimal charging of PHEVs by shaving the peak load. Meanwhile, in [16] the photovoltaic (PV) battery is used to charge the vehicle and particle swarm optimization (PSO) is used for vehicle charging management to improve computational time and efficiency. In [17], optimal planning of PHEV charging is done through a long-term bi-objective model, where wind power generation and demand side management parameters are optimized to contain the uncertainties of the load growth. A novel unidirectional V2G management control approach is introduced in [18] by focusing on multiple energy suppliers to suppress any greedy attitude from individuals. In general, current studies on charging of PHEVs do not consider technical uncertainties that may occur from charging a PHEV. Moreover, there is lack of research on fast scheduling of PHEVs considering V2G technology and renewable energy. Therefore, there is a need to develop an effective control method for charging and discharging multiple PHEV batteries.

The aim of this paper was to develop a new management control method called charging and discharging control algorithm (CDCA) for PHEVs considering V2G technology and photovoltaic generation. The purpose of the CDCA is to be used in real time smart charging and discharging of PHEVs so as to reduce the peak power demand. The control algorithm depends on several parameters which include vehicle's SOC, arrival time, departure time, battery capacity, peak load and photovoltaic power. The proposed CDCA will assist operators in making decisions as to "when and which" of the PHEVs can be activated to consume power from the grid (charging) or supply power back to grid $(\mathrm{V} 2 \mathrm{G})$, based on parameters including the preference of the vehicle owners. Prior to developing the CDCA, the SOC of the PHEV battery was first predicted by using the proposed hybrid particle swarm optimization and artificial neural network (PSO-ANN). Three input variables were considered for the artificial neural network (ANN), namely, voltage, current and temperature. The PSO technique is used to increase and enhance the efficiency and accuracy of the ANN by optimizing the number of hidden layers and the number of nodes in each hidden layer. 


\section{Application of Particle Swarm Optimization and Artificial Neural Network (PSO-ANN) for Predicting Penetration of Plug-in Electric Vehicles (PHEVs) Battery State of Charge (SOC)}

Basically, an ANN is a distributed processing system that models nonlinear systems and attempts to simulate the functionality of the human brain. Neural network has the advantage of solving complex nonlinear relationships between a system's input and output and it has the capability of solving non-linear complex functions through training and learning of the input and output of the system. Basically, an ANN consists of neurons which are simple connected elements.

For predicting the PHEV battery SOC, the multi-layer perceptron ANN model with back-propagation algorithm is applied in this study. Figure 1 shows the structure of the ANN model in which it has three input parameters, namely, voltage $(V)$, current $(I)$ and temperature $(T)$, a number of hidden layers and one output parameter which is the SOC. The ANN receives the voltage $(V)$, current $(I)$ and temperature $(T)$ as inputs and predicts the value of the battery SOC. The data used to train the ANN are collected from the NASA Center. The SOC is then calculated using the following equation:

$$
\operatorname{SOC}(i)=\operatorname{SOC}(i-1)+\frac{I(i)}{Q n} \Delta t
$$

where $I$ is the discharging current, $Q n$ is the rated battery capacity and $\Delta t$ is the change in temperature.

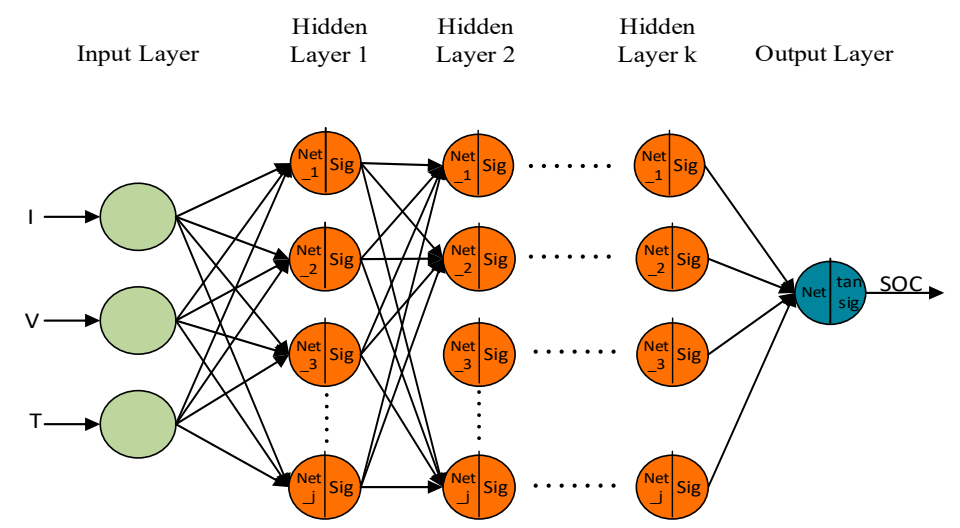

Figure 1. Artificial neural network (ANN) structure with three inputs, one output and multi hidden nodes.

All the inputs and outputs of the ANN are expressed by the following equations:

$$
\begin{gathered}
\text { Input }=\left[\begin{array}{c}
I_{1} V_{1} T_{1} \\
I_{2} V_{2} T_{2} \\
\vdots \\
I_{n} V_{n} T_{n}
\end{array}\right] \\
\text { Output }=\left[\begin{array}{c}
S O C_{1} \\
S O C_{2} \\
\vdots \\
S O C_{n}
\end{array}\right]
\end{gathered}
$$

where $I$ is the discharging current of battery, $V$ is the voltage of battery and $S O C$ is the state of charge of the battery.

In this study, the Levenberg-Marquardt back propagation algorithm has been selected for training the ANN in the Matlab toolbox. The Levenberg-Marquardt algorithm has been selected due to its minimal localization error as well as its efficiency and speed. 
To evaluate the performance of the trained ANN, the root-mean-square error (RMSE) and mean absolute error (MAE) are considered. The errors between the actual and predicted ANN outputs using the RMSE and MAE are reduced to achieve better ANN performance. The statistical indices of MAE and RMSE are given by,

$$
\begin{aligned}
M A E & =\sum_{i=1}^{n} \frac{1}{n} X\left|S O C_{A_{i}}-S O C_{p_{i}}\right| \\
R M S E & =\sqrt{\frac{1}{n} \sum_{i=1}^{n}\left(S O C_{A_{i}}-S O C_{p_{i}}\right)}
\end{aligned}
$$

where error $=S O C_{A}-S O C_{P}, S O C_{A}$ is the state of charge of the actual data, $S O C_{P}$ is the state of charge of the predicted data and $n$ is the number of samples.

\section{Implementation of Hybrid PSO-ANN for Predicting PHEV Battery SOC}

ANN architecture has significant parameters such as hidden layers, number of neurons or nodes, and learning rate (LR). The trial and error method is normally used to determine the optimum parameters, where the number of hidden layers is set to one or two for most of the time, and neurons in each hidden layer are set randomly. To overcome the time-consuming trial and error process in determining the optimum ANN parameters, the PSO is used to optimize the number of hidden layers, and the number of hidden nodes in each hidden layer. However, the LR which is in the range from 0 to 1 , is set by using an iterative method. In this work, 10 populations and 500 iterations are used to achieve minimum RMSE value. The objective functions of the hybrid PSO-ANN are based on the MAE and RMSE given in Equations (4) and (5), respectively.

In the PSO technique, two main parameters, namely, velocity and position of particles are updated by using the following equations,

$$
\begin{gathered}
V_{i}^{d}(t+1)=w V_{i}^{d}(t)+c_{1} r_{1}\left(P_{i}^{d}(t)-Z_{i}^{d}(t)\right)+c_{2} r_{2}\left(P_{i}^{d}(t)-Z_{i}^{d}(t)\right) \\
Z_{i}^{d}(t+1)=Z_{i}^{d}(t)+V_{i}^{d}(t+1)
\end{gathered}
$$

In the PSO implementation, 10 populations and 500 iterations are set to achieve minimum MAE and RMSE. Such values of population and iterations are concluded by trial and error process that leads to bests results. Figure 2 shows the flowchart of the proposed hybrid lighting search algorithm artificial neural network and artificial neural network (LSA-ANN) implementation for predicting the PHEV battery SOC.

From Figure 2, the determination of optimum number of neurons and hidden layers using the hybrid PSO-ANN is described as follows:

i. Initialize the PSO algorithm by specifying the parameters such as the population size, $\mathrm{N}=10$; number of dimensions, $\mathrm{D}=5$; number of iterations, $T=500$; max weight $=0.9$, minimum weight $=0.4$; and acceleration coefficient $c_{1}=c_{2}=2$.

ii. Randomly generates the initial positions within feasible solution combinations and obtains the initial pbest and gbest.

iii. Select the number of hidden neurons (Ne) and hidden layers (HLs) and train the ANN.

iv. Train the ANN and evaluate the fitness value by calculating the objective functions of MAE and RMSE.

v. Update the local best (pbest), the best previous position of each particles and global best (gbest) position of all particles.

vi. Update the velocity using (6) within the velocity interval of - Vmax and + Vmin.

vii. Update the particle position using (7). There are possibilities that the position is rejected. Therefore, look for other combinations. 
viii. Repeat steps (iii) and (iv).

ix. Check if the maximum population size is reached. If not, compare the pbest value with the global best (gbest), if it has a minimum value, save it as the new global best.

x. Repeat steps (v) to (ix) until reaching the stopping criterion so as to obtain the optimal hidden layers and hidden neurons.

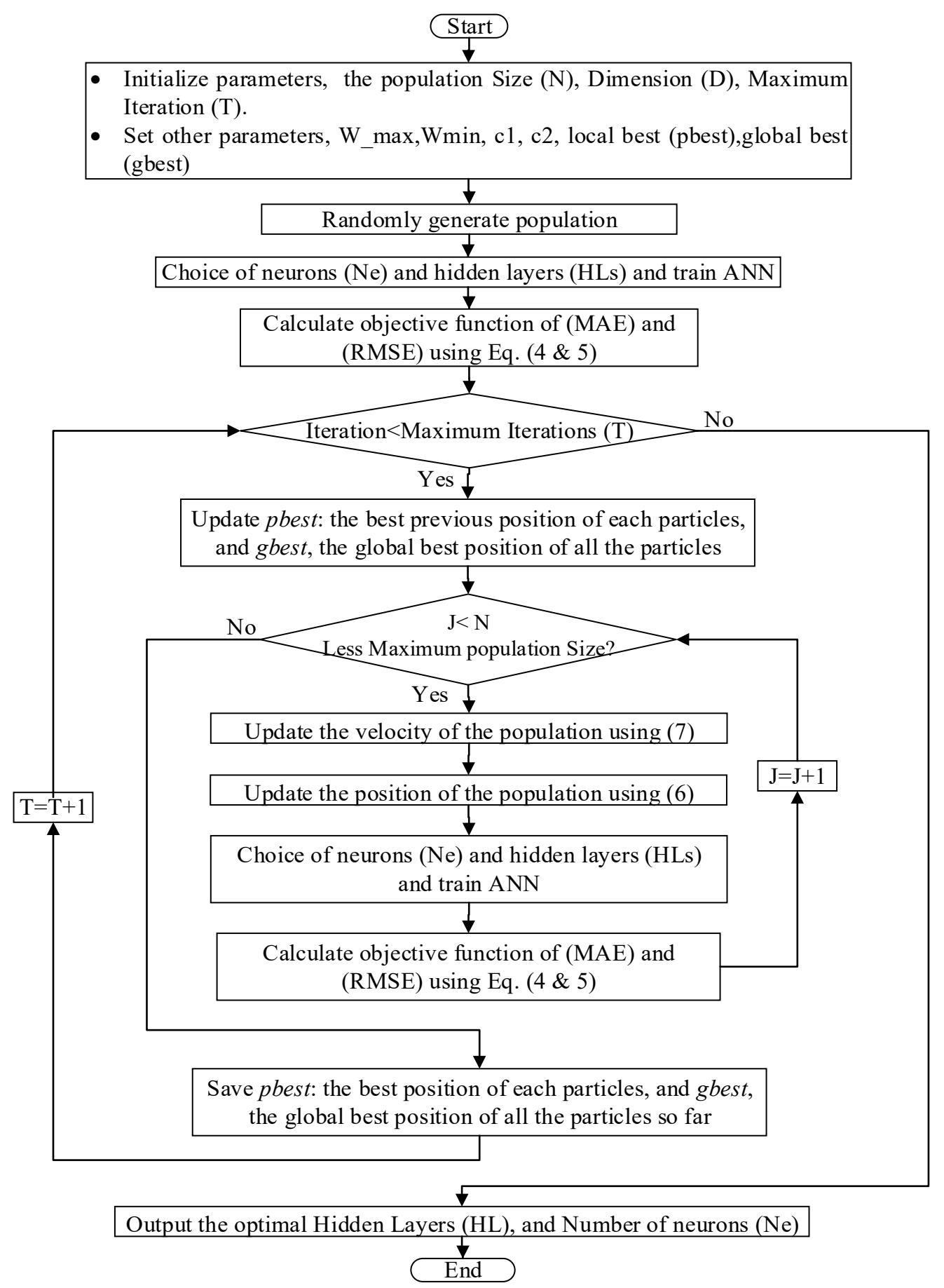

Figure 2. Flowchart of the hybrid particle swarm optimization and artificial neural network (PSO-ANN). Abbreviations: RMSE, root-mean-square error; MAE, mean absolute error; ANN, artificial neural network. 


\section{Management Control Method}

With the increasing number of PHEVs on the road, the energy demand will also be increased to charge a large number of electric vehicles. This will lead to a significant increase in load in the distribution system when PHEV batteries are being charged. In addition, if the charging process for a large number of PHEVs is not coordinated, it can overload the grid capacity and may result in system instability. Therefore, a management control algorithm called the charging and discharging control algorithm (CDCA) is proposed to coordinate both charging and discharging of PHEVs. In addition, by properly charging and discharging the PHEV batteries, smooth load consumption profile and reduced power quality impacts to the system can be obtained.

PHEVs differ in size, battery capacity and energy consumption per mile. In this project a $25 \mathrm{kWh}$ battery was used in the control system. Whenever a PHEV is connected to the parking garage, the owner of it will set the next departure time and the system will make a record of this. To not influence the driving behaviors of the PHEV owners at the departure time, the SOC of the batteries are expected to be at least $90 \%$ of its full capacity. To consider battery protection, the SOC of the PHEV's battery should not go below a certain limit. Therefore, when a certain PHEV's SOC is below a certain limit, it should not be involved in the discharging process needed in the V2G service; but during the charging process it operates as normal. By implementing the CDCA, the battery banks in the PHEVs can be effectively protected from over discharging, thus extending the lifetime of the battery banks.

\subsection{Charging Load Forecasting Model}

The determination the PHEV charging load forecasting models is classified according to time-scales or the load prediction method as three categories which are classified based on the length of the predicted time-scale, namely, short-term $(8 \mathrm{~h})$ forecasting, mid-term (day ahead) forecasting and long-term (multiple days ahead) forecasting [19]. In addition to time-scale classification, PHEV charging load forecasting models can be classified depending on the load prediction method [20]. Many researchers have used the deterministic approach which is based on load prediction using the actual vehicle $24 \mathrm{~h}$ data such as the SOC of battery [21]. Since the data pool of PHEV charging load is extremely limited so far, some parameters are manually set according to traffic data of electric vehicles. In this work, the PHEV model is represented in the power grid as a storage system, where the OpensDSS software (The Linux Foundation, San Francisco, CA, USA) has been set to a time series solution mode for a one-day load profile (15-min time interval), and the controlling of the vehicles in the power grid to be charged or discharged is done externally through Matlab software. However, since the data pool of PHEV charging load and driving patterns are extremely limited so far, the CDCA is developed based on a real time control where the battery SOC, along with the power demand, power grid and PV power output will determine the maximum allowable rate for charging the vehicles taking into consideration that no violation would occur to the network voltage profile. If any violation has existed at any specific time, the standby discharging vehicles would feedback the grid with power until the voltage profile bounced back to the permissible limit which is from 0.95 to 1.05 p.u. The approach that is used in the proposed CDCA is mainly to limit the impact of PHEVs charging on the alternating current (AC) utility grid while letting the PHEVs participate in the V2G power transactions at peak hours [22].

\subsection{Charging and Discharging Control Algorithm}

The proposed CDCA considers the arriving time and departure time of vehicle users to follow the working hours of employees which starts at 9 a.m. to 5 p.m., thus giving a total time of $8 \mathrm{~h}$. To implement a time series solution mode in the OpendDSS software, a 15 min daily load shape of a commercial area in Malaysia, shown in Figure 3, is integrated to all the distribution loads. The flowchart of the CDCA for PHEVs is shown in Figure 4. The control algorithm starts by collecting the initial data of the vehicle's battery like voltage, current, temperature and the predicted SOC from the 
trained ANN. After that, all the PHEVs would be sorted and idled for either charging or discharging process based on the level of the SOC. In the CDCA, when the vehicle battery's SOC has a capacity more than $60 \%$, the vehicle is queued for V2G discharging. However, for a SOC with a capacity less than $60 \%$, the vehicle is queued for charging based on the set priority level described in Figure 4. Voltage profile and power loss of the system are checked at each vehicle charging. If any violation occurs, the queued PHEVs which have been initialized for discharging would be utilized to supply power back to the system to return to normal without any violation that occurs during charging. The PV output power in the control system is also used as a supply source to charge vehicles during peak hours.

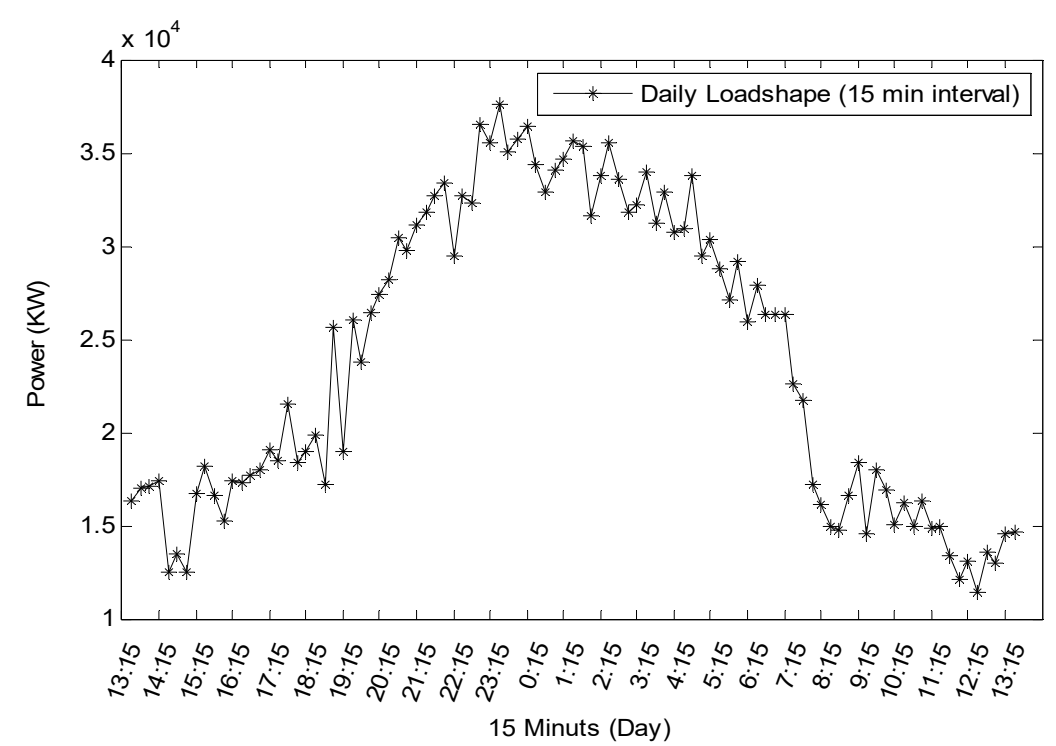

Figure 3. Daily load shape of a commercial area in Malaysia.

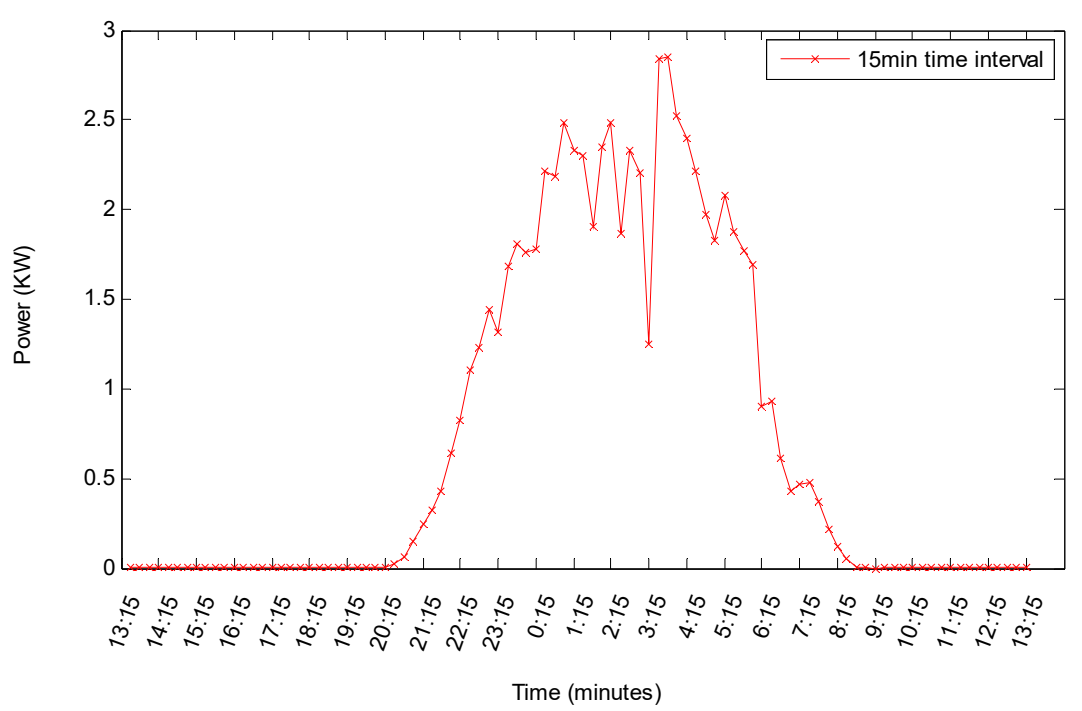

Figure 4. Photovoltaic (PV) generation output power of Universti Kebangsaan Malaysia (UKM) solar system.

The charging and discharging of PHEVs at different SOC capacities would have different priorities to be considered [23]. Considering that the PHEV in the parking garage will have a different SOC, its average constant power requirement will differ. Some PHEVs may need a huge amount of energy because of their low level SOC and therefore these PHEVs should be classified into the high priority level. For PHEV's SOC which is already high at the departure time, this kind of PHEV should be 
classified into the lower priority level. Therefore, a priority classification level (PCL) for charging a PHEV should be designed based on priority levels and it is given by,

$$
\operatorname{PCL}(i)=\left\{\begin{array}{l}
1, \text { High Priority } \\
2, \text { Medium Priority } \\
3, \text { Low Priority }
\end{array} \quad i=1,2,3, \ldots, N_{\text {vehicles }}\right.
$$

To limit the impact of PHEVs charging to the AC utility grid while letting the PHEVs participate in the V2G power transaction, a load shape of $\mathrm{PV}$ output power solar system that already installed at Universti Kebangsaan Malaysia (UKM) is integrated in the developed CDCA, where it represents the PV power output with 15-min time interval as shown in Figure 4. So, to smooth the charging process of the large scale of PHEVs, the parking garage should have a smart charging algorithm that can adjust the charging and discharging rates for the PHEVs based on utility with the assistance of PV generation output power on peak hours.

Thus, the proposed CDCA shown in Figure 5 considers technical parameters such as AC power flow (Pgrid), and PV output power (PPV), arriving time (AT), departure time (DT), power demand (PD), SOC of PHEV battery, and priority level (PrLevel). These parameters are considered to determine the availability of PHEVs to be charged, idle or discharged. System constraints such as bus voltage magnitude and power losses in the system are also considered. To maintain power system voltage security, the bus voltage magnitudes are kept within permissible limit as depicted in the following expression:

$$
0.95 \leq V_{j} \leq 1.05 \quad j=1,2,3, \ldots, N_{b u s}
$$

where $V_{j}$ is the root mean square (RMS) value of the $i$ th bus voltage.

The total power loss which that may occur during PHEV charging is expressed as follows:

$$
C P_{\text {loss }}=\sum_{i=1}^{n} C P_{\text {loss }} i=1,2,3, \ldots, n
$$

where $n$ is the number of lines in the system.

The procedures for implementing the proposed CDCA are described as follows:

i. Obtain input network information of the test distribution systems such as bus, line and load data.

ii. Obtain the PHEV battery characteristics, like voltage, current and temperature, to be later controlled upon arriving at the parking lots.

iii. Initialize OPENDSS start-up in MATLAB and initialize different daily load profiles to the system.

iv. Run OpenDSS daily load profile using MATLAB and run power flow to obtain the total power loss and voltage magnitude.

v. Predict the SOC of vehicles using the developed ANN prediction model.

vi. Sort the vehicles such that the vehicles with a SOC less than $60 \%$ are charged first, and other vehicles with SOC $>60 \%$ are put on standby. When backup power sources are needed during the charging process, PHEVs with SOC $>60 \%$ will start the discharging V2G process.

vii. Power demand is verified first, such that if it is not on peak demand, the grid power is used as the charging power supply to the vehicles, otherwise PV power output is activated to provide power to PHEVs for charging.

viii. Prioritize the vehicle to three levels: high priority, medium priority and low priority. Charging starts with high priority level when SOC is less than $30 \%$, and then considers medium priority, when SOC is in the range of $30 \%-45 \%$, and lastly low priority when SOC is in the range of $45 \%-60 \%$.

ix. Run OpenDSS power flow using the MATLAB software to obtain the updated total power loss and voltage magnitude. 
x. Check the bus voltage magnitude and total power loss constraints, if both do not exceed their limits, consider the other vehicles in the queue and repeat steps from (vii) to (ix).

xi. Set V2G variable to 1, when there is a violation on the threshold value of both voltage magnitude and power loss.

xii. Start discharging the first vehicle in the queue to limit the impact of PHEVs on the voltage profile and power loss such that the voltage and power loss are within permissible limits.

xiii. Run power flow using the OpenDSS to obtain the new updated values of voltage magnitude and power loss.

xiv. Check for violations in the voltage magnitude and power loss constraints, if violation exists repeat steps (xi) to (xiii).

xv. Go to step (x) to take another vehicle from the queue for charging process.

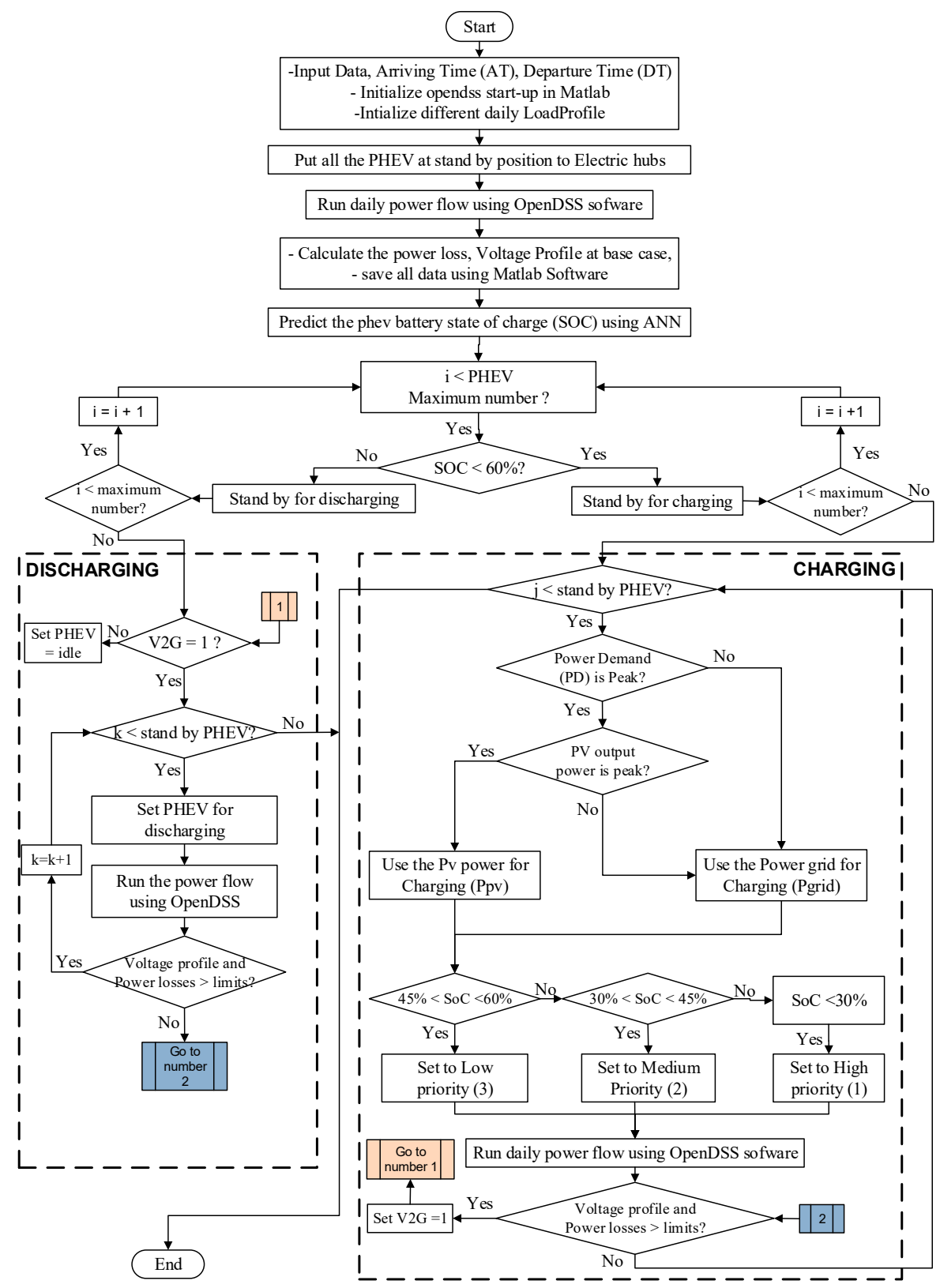

Figure 5. Flowchart of the proposed charging and discharging control algorithm. 


\section{Results and Discussion}

This section presents the results on the prediction of the SOC of the PHEV battery using the hybrid PSO-ANN and charging and discharging of PHEVs using the proposed CDCA considering the V2G energy transfer. Simulations were carried out on the Institute of Electric and Electronic Engineer (IEEE) 37 bus test distribution system and the Malaysian load profile has been considered in modeling the distribution network.

\subsection{Results of Hybrid PSO-ANN for Predicting Battery SOC of PHEV}

To enhance and improve ANN performance and create a coherent model for the prediction of PHEV's battery SOC, the PSO optimization technique is used to search for the optimal value of three main parameters in the ANN, namely, learning rate (LR), number of neurons (Ne) in each hidden layer, and finally the number of hidden layers (HLs). Different population sizes are implemented to permit the PSO to choose and select the population size that could give the minimum error and computation time. As a consequence, the minimum objective function or fitness value can be achieved by selecting the most appropriate number of population sizes to assist in improving the ANN performance during training and testing. The objective function that is considered for optimization is the RMSE. The obtained RMSE values at 10, 20,30 and 40 population sizes are shown in Figure 6. The best number of population size $(\mathrm{N})$ for optimizing the three parameters, namely, LR, Ne and HLs in the hybrid PSO-ANN is 20, in which the PSO algorithm gives the lowest fitness value of the RMSE compared to other population sizes 10, 30 and 40. The best fitness value of RMSE acquired in PSO-ANN at a population size of 20 is $0.9320 \times 10^{-3}$ after 79 iterations. Table 1 shows the optimum learning rate (LR), number of neurons in the hidden layers (Ne1, Ne2, Ne3) and number of hidden layers (HLs), obtained by using the hybrid PSO-ANN technique considering different population sizes. From the table, the PSO-ANN optimum values acquired for the LR, HL, Ne1 and Ne2 are 0.3663, 3, 13, 13 and 10, respectively. The best value of the optimized four parameters are indicated in bold at a population size of 20. Table 2 illustrates three statistical indices, namely, RMSE, mean square error (MSE) and MAE to evaluate the ANN accuracy at the optimized parameters found.

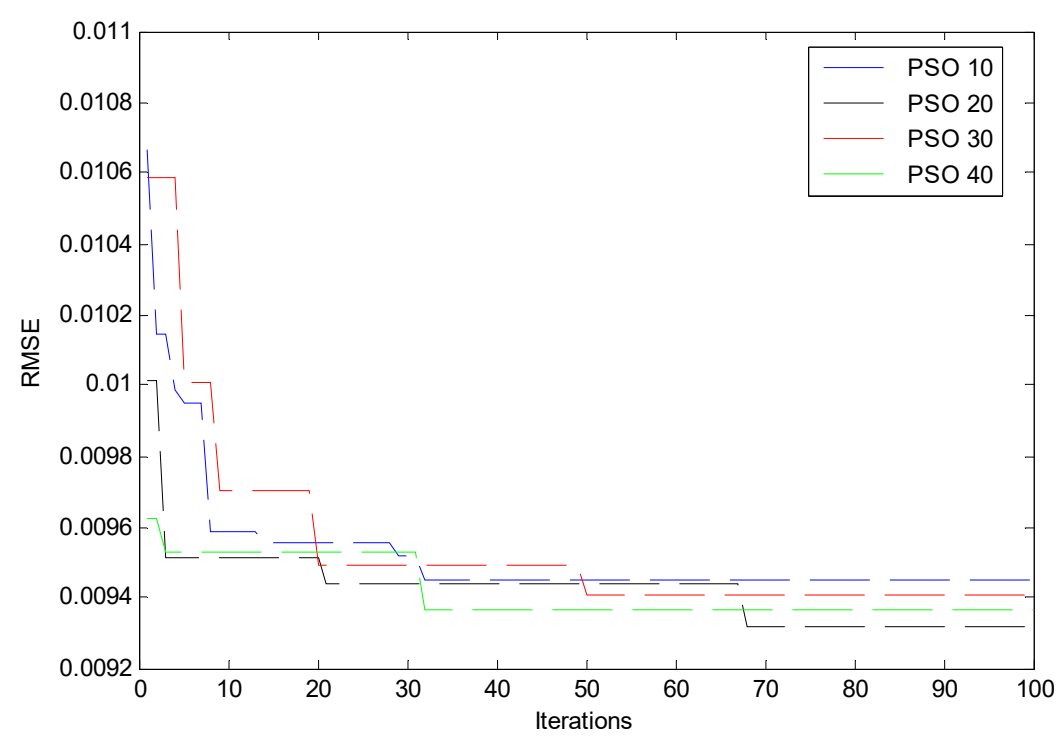

Figure 6. RMSE value of the hybrid PSO-ANN at different population sizes. 
Table 1. Optimum value of optimized parameters in hybrid particle swarm optimization and artificial neural network (PSO-ANN) at different population sizes.

\begin{tabular}{ccc}
\hline Population Size & Parameters & Hybrid PSO-ANN \\
\hline \multirow{3}{*}{10} & Learning rate (LR) & 0.7195 \\
& Hidden Layers & 3 \\
& Neurons (Ne)1 & 14 \\
& Ne2 & 9 \\
Ne3 & 8 \\
\hline \multirow{2}{*}{20} & LR & 0.3663 \\
& Hidden Layers & 3 \\
& Ne1 & 13 \\
& Ne2 & 13 \\
Ne3 & 10 \\
\hline \multirow{2}{*}{30} & LR & 0.5645 \\
& Hidden Layers & 3 \\
& Ne1 & 8 \\
& Ne2 & 20 \\
& Ne3 & 7 \\
\hline & LR & 0.9667 \\
& Hidden Layers & 3 \\
& Ne1 & 12 \\
& Ne2 & 13 \\
& Ne3 & 1 \\
\hline
\end{tabular}

Table 2. Statistical indices at different population sizes in hybrid PSO-ANN.

\begin{tabular}{ccccc}
\hline \multirow{2}{*}{ Parameter } & \multicolumn{4}{c}{ Population Size } \\
\cline { 2 - 5 } & $\mathbf{1 0}$ & $\mathbf{2 0}$ & $\mathbf{3 0}$ & $\mathbf{4 0}$ \\
\hline Root-mean-square error (RMSE) (\%) & 0.9451 & 0.9320 & 0.9408 & 0.9368 \\
Mean square error (MSE) (\%) & 0.0089 & 0.0087 & 0.0089 & 0.0088 \\
Mean absolute error (MAE) (\%) & 0.6349 & 0.6306 & 0.6344 & 0.6478 \\
\hline
\end{tabular}

The target test data or actual data and the predicted data of the battery SOC by using the hybrid PSO-ANN is shown in Figure 7. The SOC estimation is represented by the red line, while the actual data of the SOC is represented by the blue line. The actual and estimated SOC is standardized in the range from $90 \%$ to $10 \%$. The validation between the estimated and actual SOC of the battery has also been carried out and the results show that the estimated SOC is nearly aligned with the actual SOC with some fluctuation noticed in Figure 7. The slight fluctuations of the estimated values compared with the actual ones are noticed with maximum SOC error percentage of $5.2178 \%$ as shown in Figure 8. The fluctuation that occurs in the estimated SOC are mainly caused due to the less input parameter numbers of the ANN model; however, considering more input parameters would increase the reliability of the model. The regression coefficient $(\mathrm{R})$ is a good indicator for evaluating the estimation performance of PSO-ANN. As shown in Figure 9, the regression coefficient of PSO-ANN algorithms is 0.99915. The regression coefficient results are in close agreement to unity which validates the accuracy of the PSO-ANN model. 


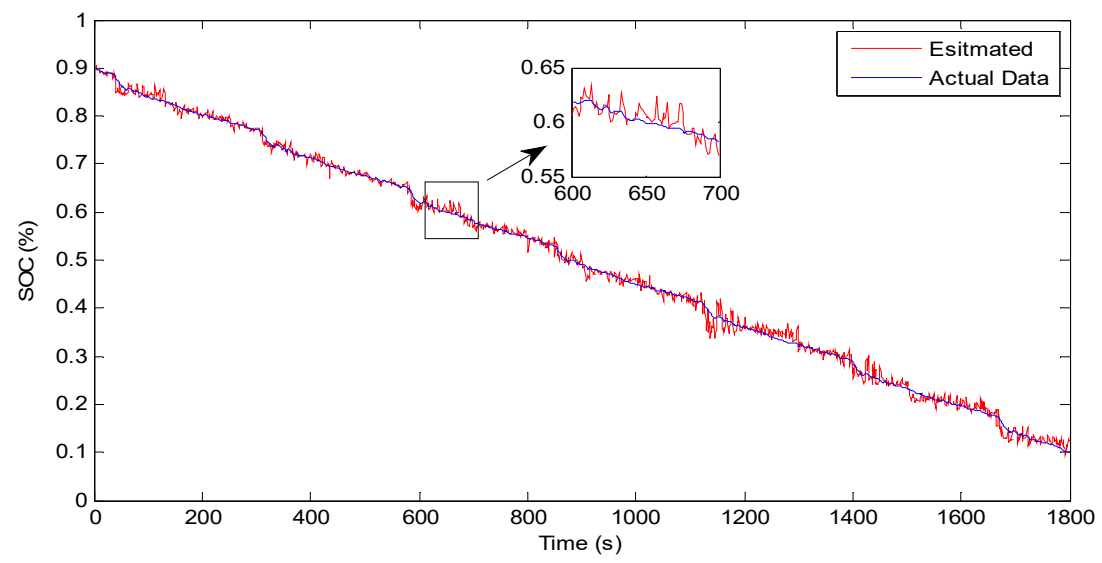

Figure 7. State of charge (SOC) estimated data values compared with actual data for PSO-ANN.

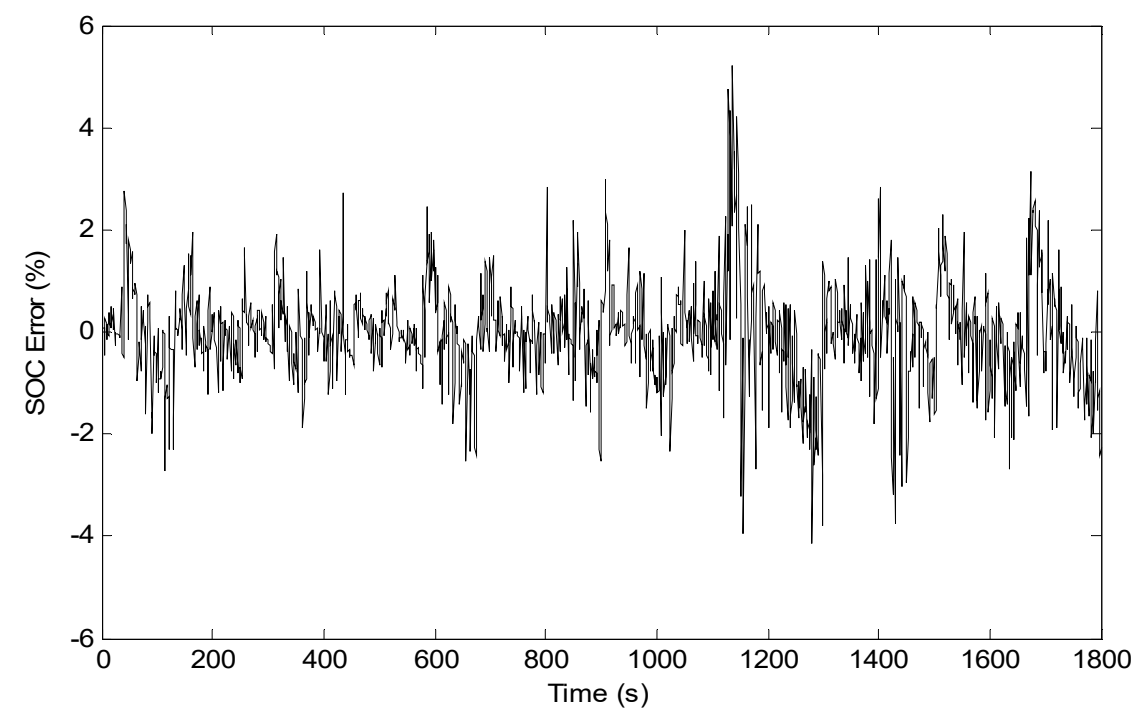

Figure 8. Error percentage obtained from estimated and actual data of SOC for PSO-ANN algorithm.

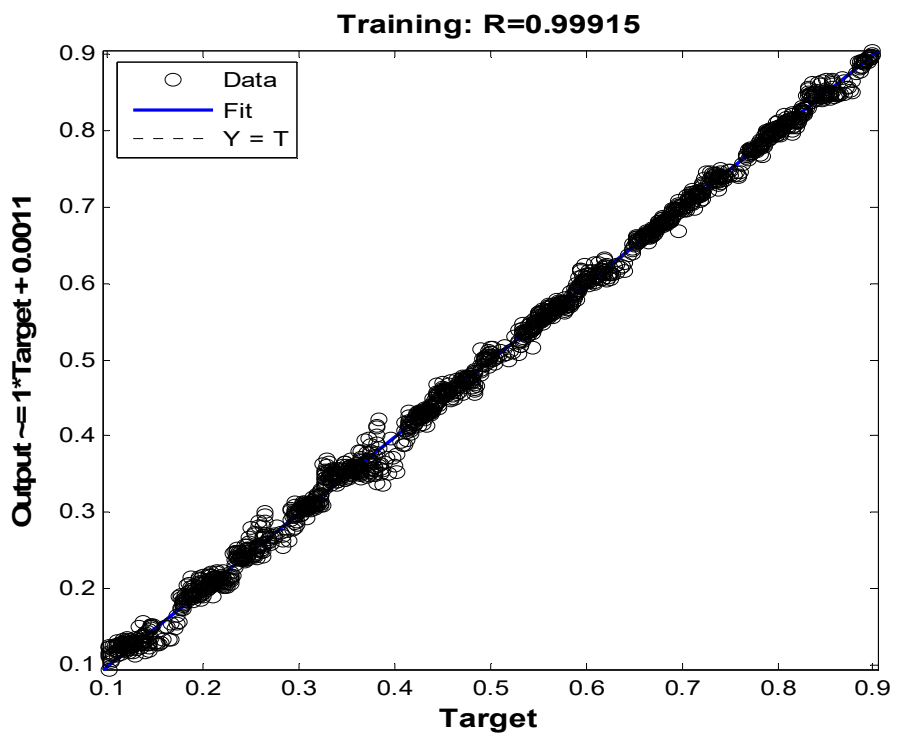

Figure 9. Regression plot of LSA-ANN. 


\subsection{Results of Charging and Discharging Control Algorithm of PHEVS}

The proposed CDCA to coordinate the charging and discharging of PHEVs in the distribution system has been implemented in time series solution mode by using the OpenDSS. The whole process of control is done in MATLAB, where the set value of the $\mathrm{V} 2 \mathrm{G}$ variable determines the charging or discharging of the PHEVs. A daily Malaysian load shape with a 15 min time interval was integrated in each load of the distribution system. The SOC of PHEVs are firstly estimated by the hybrid PSO-ANN model to determine the vehicles SOC during arrival time. The CDCA is mainly coordinating the queued PHEVs for charging and discharging, where vehicles that need to be charged (SOC $\leq 60 \%$ ), are prioritized. If any violation occurs in the voltage profile of the system, vehicles with SOC greater than $60 \%$ are activated, to support the distribution system with V2G technology.

The battery life cycle is considered in the CDCA where the maximum allowable charging and minimum discharging value are $90 \%$ and $10 \%$, respectively. The estimated SOC of vehicles are firstly introduced as shown in Figure 10. The battery SOC value is selected from highest priority level (SOC $<35 \%)$, followed by second highest priority level $(35 \%<$ SOC $<45 \%)$, and lastly with third highest priority level $(45 \%<$ SOC $<60 \%)$.

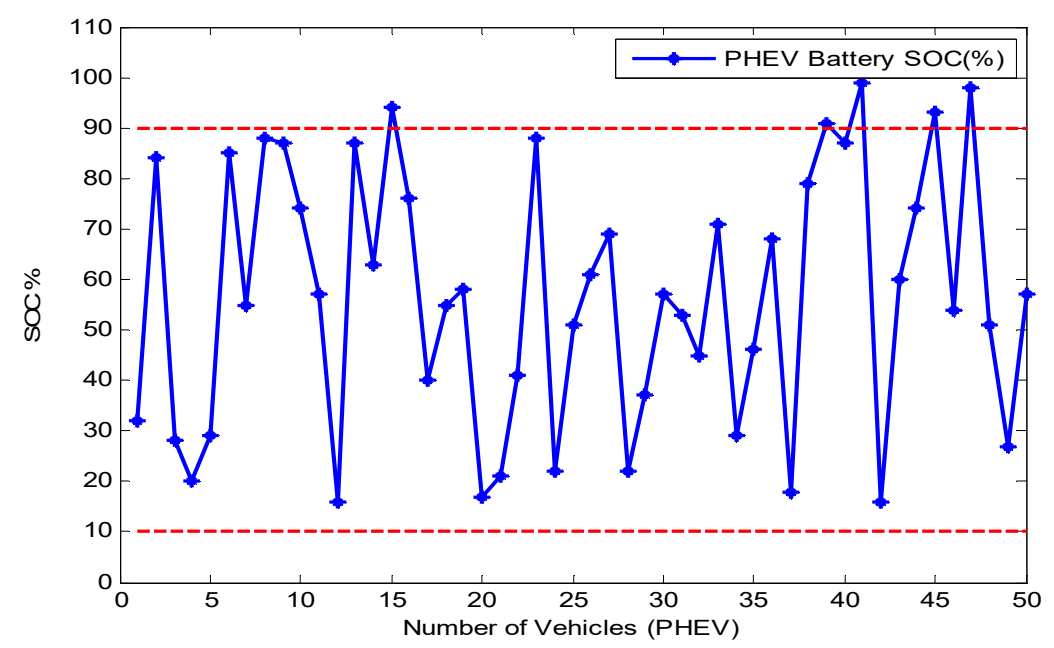

Figure 10. Battery SOC of various vehicles.

Table 3 shows the number of vehicles with SOC set below the limit as in the proposed algorithm and all these vehicles are sorted aside for the charging process. The properties are stored such as the maximum kWh battery, the current SOC of the vehicles that that has been estimated by the ANN model, parking type and hours duration which is the difference between the arriving times (AT) and the departure time (DT). The vehicles with a SOC above the permissible limit set in the proposed algorithm are sorted with all their properties for the discharging process shown in Table 4. All the queued vehicles listed in the table are in idling state and the vehicles are sorted based on the SOC value of vehicles, where the vehicle with highest priority would be charged first and followed by least priority level and so on. If any violation occurs in the voltage profile of the system during the process of charging the vehicles listed in Table 3, one vehicle at a time would be activated to discharging mode to support the grid and shift the voltage profile to its permissible limit as set in the proposed CDCA.

The voltage profile at the base case is firstly investigated in the test IEEE 37 bus distribution system. Figure 11 depicts the voltage profile of all the buses, in which the voltage values of all buses are within the permissible limit of the IEEE standard which ranges from 0.95 to 1.05 p.u. From another aspect, the voltage profile of the bus where assumedly the charging station is located at bus 735 is also investigated; the solution mode is set to time series mode with 15 min time intervals as shown in Figure 12. The base case power loss is investigated as well with $15 \mathrm{~min}$ time interval solution mode 
as shown in Figure 13. From the figure, the maximum power loss of the system reaches $115 \mathrm{~kW}$ at 6:30 p.m.

Table 3. Plug-in electric vehicle (PHEV) storage charging characteristics.

\begin{tabular}{|c|c|c|c|c|c|c|}
\hline Vehicle & Bus Name & $\begin{array}{l}\text { Stored } \\
(\mathbf{k W h})\end{array}$ & $\begin{array}{l}\text { Stored } \\
(\text { SOC \%) }\end{array}$ & Enabled & Parking Type & Duration (h) \\
\hline PHEV12 & 735 & 50 & 16 & Charging & Commercial & 1 \\
\hline PHEV42 & 735 & 50 & 16 & Charging & Governmental & 8 \\
\hline PHEV20 & 735 & 50 & 17 & Charging & Governmental & 8 \\
\hline PHEV37 & 735 & 50 & 18 & Charging & Commercial & 6 \\
\hline PHEV4 & 735 & 50 & 20 & Charging & Governmental & 8 \\
\hline PHEV21 & 735 & 50 & 21 & Charging & Governmental & 8 \\
\hline PHEV24 & 735 & 50 & 22 & Charging & Commercial & 5 \\
\hline PHEV28 & 735 & 50 & 22 & Charging & Commercial & 4 \\
\hline PHEV49 & 735 & 50 & 27 & Charging & Governmental & 8 \\
\hline PHEV3 & 735 & 50 & 28 & Charging & Governmental & 8 \\
\hline PHEV5 & 735 & 50 & 29 & Charging & Commercial & 4 \\
\hline PHEV34 & 735 & 50 & 29 & Charging & Commercial & 3 \\
\hline . & . & . & . & . & . & . \\
\hline . & . & . & . & . & . & . \\
\hline . & . & . & . & . & . & . \\
\hline . & . & . & . & . & . & . \\
\hline PHEV43 & 735 & 50 & 60 & Charging & Governmental & 8 \\
\hline
\end{tabular}

Table 4. PHEV storage discharging characteristics.

\begin{tabular}{ccccccc}
\hline Vehicle & Bus Name & $\begin{array}{c}\text { Stored } \\
\mathbf{( k W h )}\end{array}$ & $\begin{array}{c}\text { Stored } \\
(\mathbf{S O C} \mathbf{\%})\end{array}$ & Enabled & Parking Type & Duration (h) \\
\hline PHEV41 & 735 & 50 & 99 & Idling & Governmental & 8 \\
PHEV47 & 735 & 50 & 98 & Idling & Commercial & 2 \\
PHEV15 & 735 & 50 & 94 & Idling & Commercial & 3 \\
PHEV45 & 735 & 50 & 93 & Idling & Governmental & 8 \\
PHEV39 & 735 & 50 & 91 & Idling & Governmental & 8 \\
PHEV8 & 735 & 50 & 88 & Idling & Commercial & 6 \\
PHEV23 & 735 & 50 & 88 & Idling & Governmental & 8 \\
PHEV9 & 735 & 50 & 87 & Idling & Governmental & 8 \\
PHEV13 & 735 & 50 & 87 & Idling & Commercial & 8 \\
PHEV40 & 735 & 50 & 87 & Idling & Commercial & 6 \\
PHEV6 & 735 & 50 & 85 & Idling & Governmental & 8 \\
PHEV2 & 735 & 50 & 84 & Idling & Governmental & 8 \\
. &. &. &. &. & &. \\
. &. &. &. &. & &. \\
PHEV26 & 735 & 50 & 61 & Idling & Governmental & 8 \\
\hline
\end{tabular}

Tables 5-7 show the charging process of one random selected vehicle with highest priority level, medium priority level and low priority level, respectively. The control algorithm would check the voltage profile of the distribution system at each $15 \mathrm{~min}$ interval. The consumption of power from the grid is $25 \mathrm{~kW}$ and the maximum value of battery stored energy is set to $50 \mathrm{kWh}$. In Table 5, the selected vehicle number 24 is considered the highest priority level since the battery SOC is below $35 \%$. It is shown that the voltage profile at 15 min charging time solution is within its permissible limit, and the charging time to charge the maximum charging capacity of the vehicle battery ( $50 \mathrm{kWh}$ ) is almost 1 $\mathrm{h}$ and $45 \mathrm{~min}$. Similarly, the voltage profile of the vehicles with medium and low priority levels are within the limit and so no voltage violation occurs at this point of time as shown in Tables 6 and 7 , respectively. However, the duration to charge the vehicles at medium and low priority levels are $1 \mathrm{~h}$ 
$30 \mathrm{~min}$ and $1 \mathrm{~h} 15 \mathrm{~min}$, respectively. It is noted from the tables that the charging duration is directly proportional to the priority level of the vehicles. At the highest priority level, much longer time is needed to fully charge the battery.

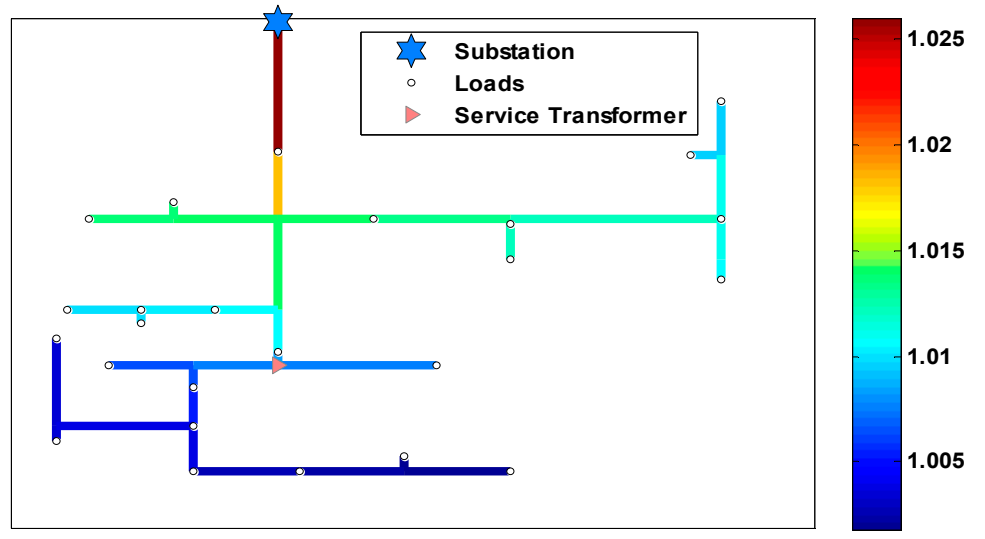

Figure 11. Voltage profile of all buses in the IEEE 37 bus distribution system.

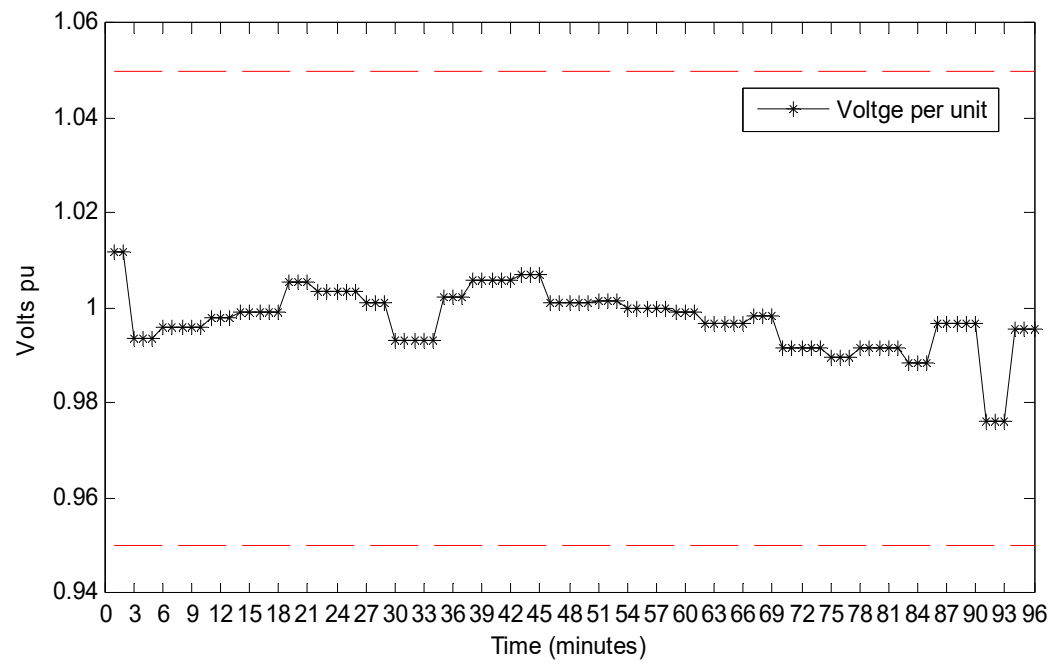

Figure 12. Voltage profile of bus 735 over 15 min time intervals.

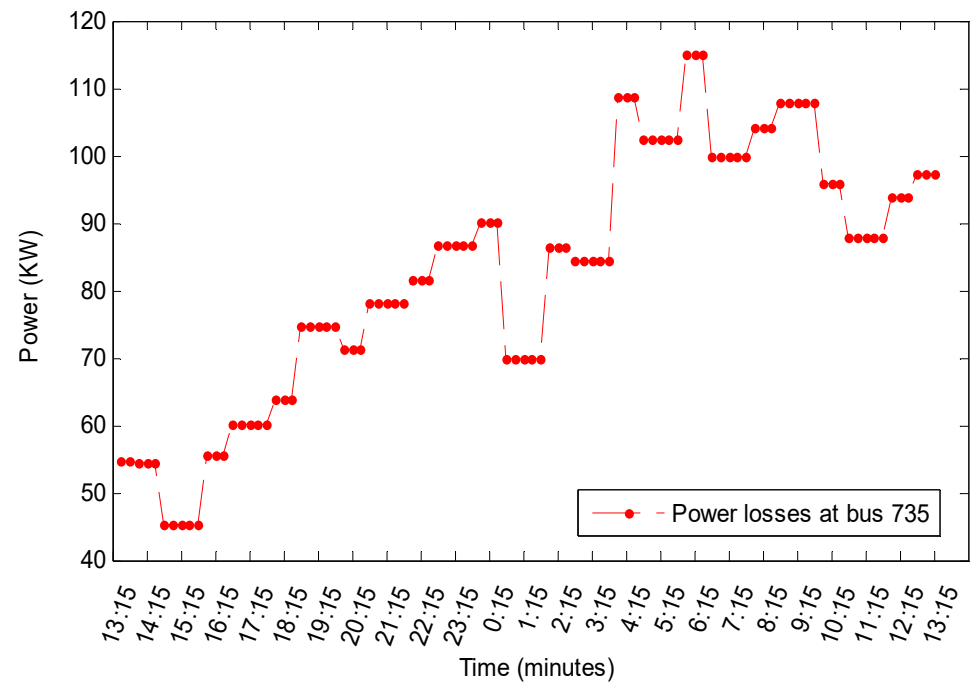

Figure 13. Losses at bus 735 at base case over $24 \mathrm{~h}$ (15 min intervals). 
Table 5. Charging characteristics of a selected vehicle with a high priority level.

\begin{tabular}{cccccccc}
\hline Vehicle & Bus Name & $\begin{array}{c}\text { Stored } \\
\mathbf{( k W h )}\end{array}$ & $\begin{array}{c}\text { Stored } \\
\mathbf{( S O C} \%)\end{array}$ & State & $\begin{array}{c}\text { Time } \\
\mathbf{( m i n )}\end{array}$ & $\mathbf{k W}$ & $\begin{array}{c}\text { Voltage } \\
\mathbf{( p . u )}\end{array}$ \\
\hline & & 16.4642 & 32.9284 & Charging & 15 & 25 & 0.9794 \\
& 21.9284 & 43.8569 & Charging & 15 & 25 & 0.9794 \\
Storage.phev24 & \multirow{2}{*}{735} & 27.3926 & 54.7852 & Charging & 15 & 25 & 0.9787 \\
& & 32.8567 & 65.7135 & Charging & 15 & 25 & 0.9787 \\
& & 38.3209 & 76.6417 & Charging & 15 & 25 & 0.9787 \\
& 43.7841 & 87.5683 & Charging & 15 & 25 & 0.9813 \\
& 49.2474 & 98.4948 & Charging & 15 & 25 & 0.9813 \\
& 50 & 100 & Idling & 15 & 25 & 0.9813 \\
\hline
\end{tabular}

Table 6. Charging characteristics of a selected vehicle with a medium priority level.

\begin{tabular}{cccccccc}
\hline Vehicle & Bus Name & $\begin{array}{c}\text { Stored } \\
\mathbf{( k W h )}\end{array}$ & $\begin{array}{c}\text { Stored } \\
\mathbf{( S O C} \%)\end{array}$ & State & $\begin{array}{c}\text { Time } \\
(\mathbf{m i n})\end{array}$ & $\mathbf{k W}$ & $\begin{array}{c}\text { Voltage } \\
\mathbf{( p . u )}\end{array}$ \\
\hline & 21.4643 & 42.9286 & Charging & 15 & 25 & 0.9792 \\
& 26.9286 & 53.8572 & Charging & 15 & 25 & 0.9792 \\
Storage.phev1 & \multirow{2}{*}{735} & 32.3928 & 64.7856 & Charging & 15 & 25 & 0.9785 \\
& & 37.857 & 75.714 & Charging & 15 & 25 & 0.9785 \\
& 43.3212 & 86.6424 & Charging & 15 & 25 & 0.9785 \\
& & 48.7845 & 97.569 & Charging & 15 & 25 & 0.9811 \\
& 50 & 100 & Idling & 15 & 25 & 0.9811 \\
\hline
\end{tabular}

Table 7. Charging characteristics of a selected vehicle with a low priority level.

\begin{tabular}{cccccccc}
\hline Vehicle & Bus Name & $\begin{array}{c}\text { Stored } \\
\mathbf{( k W h )}\end{array}$ & $\begin{array}{c}\text { Stored } \\
(\mathbf{S O C} \%)\end{array}$ & State & $\begin{array}{c}\text { Time } \\
(\mathbf{m i n})\end{array}$ & $\mathbf{k W}$ & $\begin{array}{c}\text { Voltage } \\
\text { (p.u) }\end{array}$ \\
\hline & & 27.9643 & 55.9287 & Charging & 15 & 25 & 0.9791 \\
Storage.phev32 & \multirow{2}{*}{735.4287} & 66.8573 & Charging & 15 & 25 & 0.9791 \\
& & 38.8929 & 77.7858 & Charging & 15 & 25 & 0.9783 \\
& & 44.3572 & 88.7143 & Charging & 15 & 25 & 0.9783 \\
& & 49.8214 & 99.6428 & Charging & 15 & 25 & 0.9783 \\
& & 50 & 100 & Idling & 15 & 25 & 0.9809 \\
\hline
\end{tabular}

Figure 14 shows a comparison of the voltage profiles when charging control is not applied (dumb charging). It is shown that the voltage profile has exceeded its permissible limit starting from 8:15 a.m. up to almost 12:15 p.m. The dumb charging of vehicles would add extra burden to the power system. However, with the integration of PHEV in the market, the technology of V2G is an essential option to be exploited to shift back the voltage profile within the acceptable range by discharging the queued vehicles with high SOC values. Figure 15 illustrates the power losses of the distribution system under two cases, first at base case and second at dumb charging (without control). The overall power losses have significantly increased when there is no coordination on the charging process of the vehicles with almost 23\% increase. Figure 16 shows the line loading base case in the lines of the distribution system, in which the base case is compared with no coordination control line loading results of PHEV charging. The results reveal a slight increase in the thermal line loading of the transmission lines due to dumb charging.

Table 8 shows the control that was implemented to shift the under voltage resulted from excessive charging of the vehicles. It is noted that voltage violation occurs from 11:30 p.m. to 12:30 as shown in Figure 14. At that point of time, the CDCA would inject one of the discharging standby vehicles to support the grid with power through the V2G technology. As a result of injecting power to the grid, the voltage profile returns to its permissible limit with no voltage violation occurring in the system. Figure 17 shows the voltage profile after implementing the proposed CDCA. It is noticeable that the voltage in p.u, at the time of violation as depicted in Figure 14, is corrected through utilizing 
the technology of V2G on the standby discharging vehicles, by considering the lifetime of the batteries, with a boundary setting of $10 \%$ as a minimum value for the SOC.

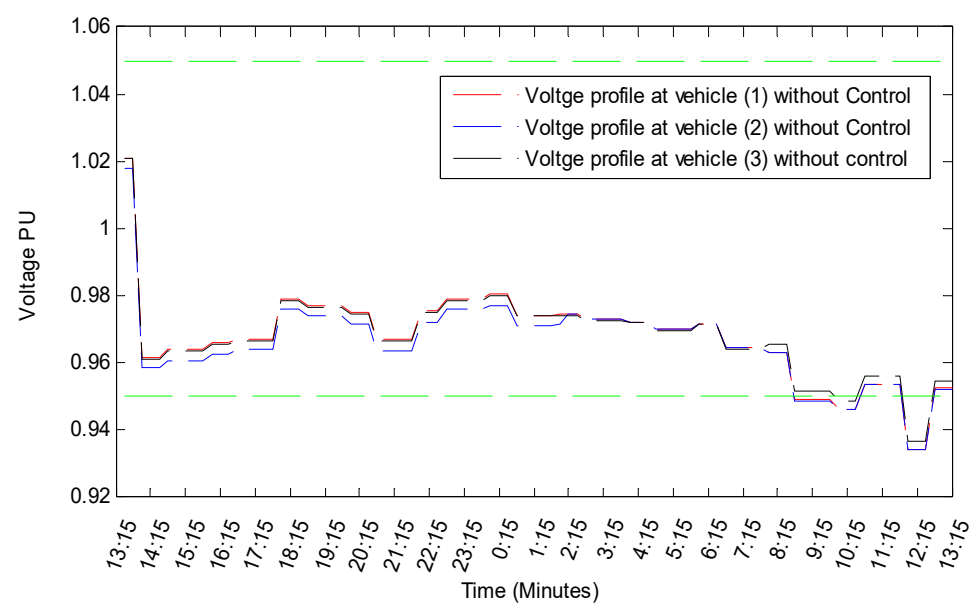

Figure 14. Voltage per unit of three different vehicles without charging control.

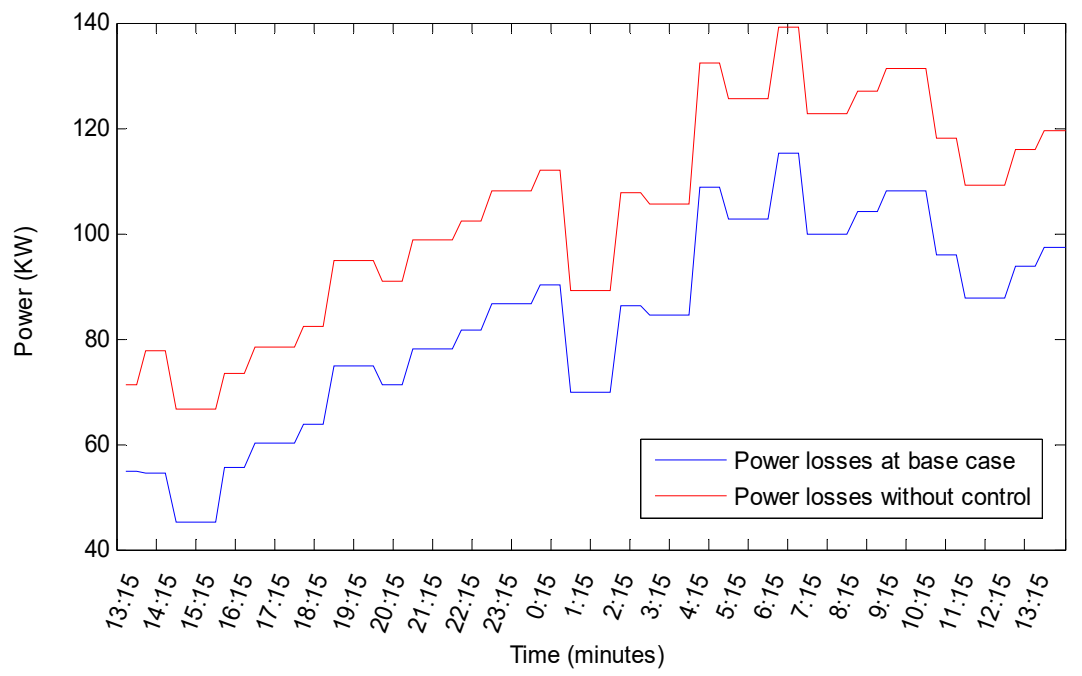

Figure 15. Power losses at base case and without charging control (dumb charging).

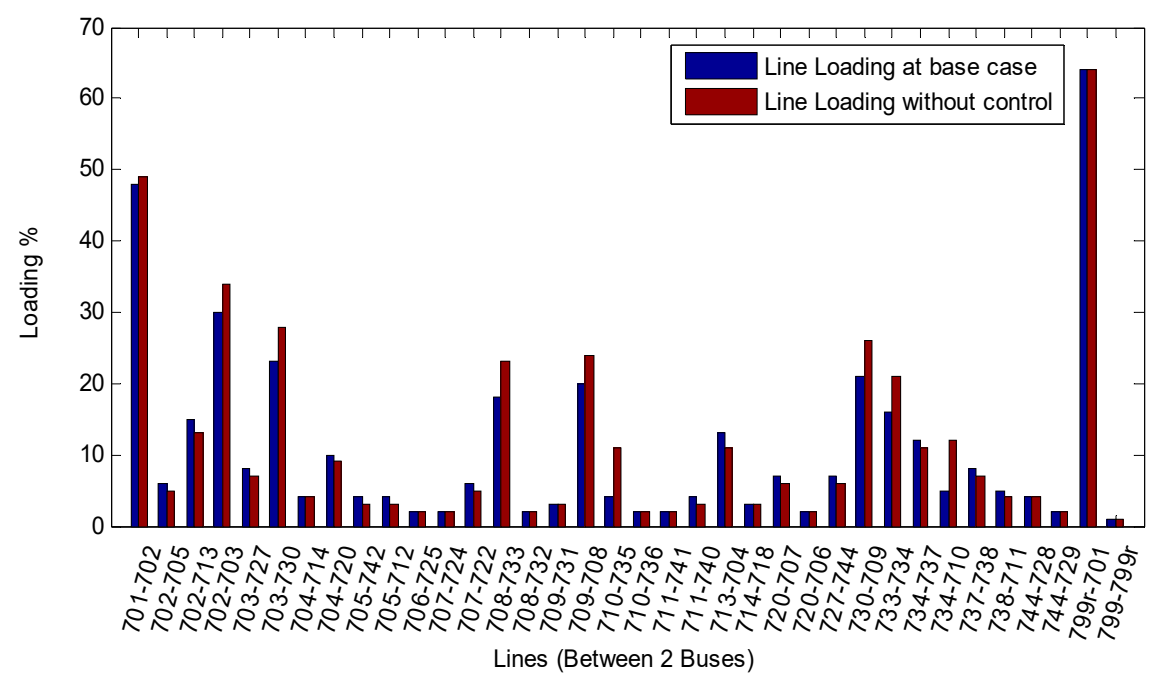

Figure 16. Line loading at base case and without charging control (dumb charging). 
Table 8. Vehicles discharging data through ehicle-to-grid (V2G) technology.

\begin{tabular}{|c|c|c|c|c|c|c|c|}
\hline Vehicle & Bus Name & $\begin{array}{l}\text { Stored } \\
(\mathrm{kWh})\end{array}$ & $\begin{array}{l}\text { Stored } \\
\text { (SOC \%) }\end{array}$ & State & $\begin{array}{l}\text { Time } \\
(\mathrm{min})\end{array}$ & $\mathbf{k W}$ & $\begin{array}{c}\text { Voltage } \\
\text { (p.u) }\end{array}$ \\
\hline \multirow{6}{*}{ Storage.phev2 } & \multirow{6}{*}{735} & 27.9643 & 83.8567 & Discharging & 15 & 25 & 1.0400 \\
\hline & & 33.4287 & 67.7134 & Discharging & 15 & 25 & 1.0400 \\
\hline & & 38.8929 & 45.5705 & Discharging & 15 & 25 & 0.9789 \\
\hline & & 44.3572 & 32.4277 & Discharging & 15 & 25 & 0.9789 \\
\hline & & 49.8214 & 19.2848 & Discharging & 15 & 25 & 0.9789 \\
\hline & & 50 & 10 & Idling & 15 & 25 & 0.9815 \\
\hline
\end{tabular}

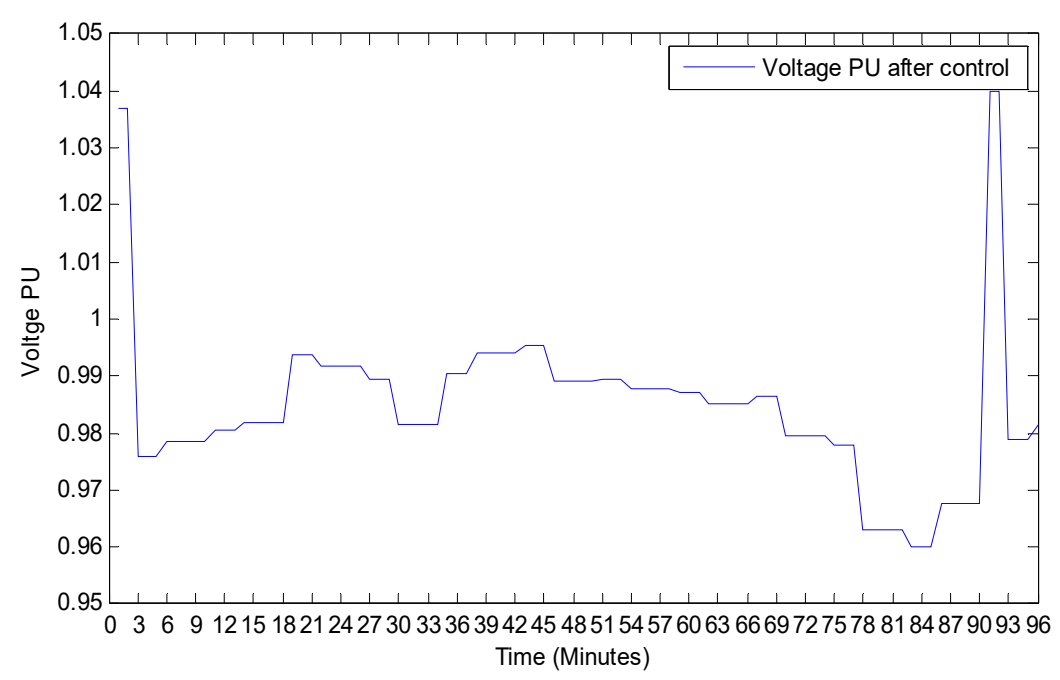

Figure 17. Voltage profile when control algorithm is introduced.

\section{Conclusions}

A new technique for predicting the SOC of a PHEV has been developed by using the hybrid PSO-ANN technique and a novel management control method called the CDCA which was developed to coordinate both charging and discharging of PHEVs. Validation between the predicted and actual power consumption of the PHEV battery SOC has been carried and the results show that the hybrid PSO-ANN gives accurate prediction with an RMSE of $0.9320 \%$. Results of the proposed CDCA indicates better performance compared to uncoordinated charging method of vehicles by considering fast charging, PV generation during peak load and V2G technology.

Author Contributions: Methodology, validation, formal analysis, investigation, writing-original draft preparation, writing - review and editing were performed by A.A. (Ahmed Aljanad), A.M., T.K., A.A. (Afida Ayob) and H.S.

Funding: The authors gratefully acknowledge the financial support provided by the University Kebangsaan Malaysia under research grants GUP-2014-072 and GP-K005302.

Conflicts of Interest: The authors declare no conflict of interest.

\section{References}

1. Salah, F.; Llg, J.P.; Flath, C.M.; Basse, H.; Van Dinther, C. Impact of electric vehicles on distribution substations: A Swiss case study. Appl. Energy 2015, 137, 88-96. [CrossRef]

2. Jason, T.; Maitra, A.; Alexander, M.; Brooks, D.; Duvall, M. Evaluation of the impact of plug-in electric vehicle loading on distribution system operations. In Proceedings of the IEEE Power and Energy Society General Meeting, Calgary, AB, Canada, 26-30 July 2009; pp. 1-6.

3. Poullikkas, A. Sustainable options for electric vehicle technologies. Renew. Sustain. Energy Rev. 2015, 41, 1277-1287. [CrossRef] 
4. Rottondi, C.; Fontana, S.; Verticale, G. Enabling privacy in vehicle-to-grid interactions for battery recharging. Energies 2014, 7, 2780-2798. [CrossRef]

5. Dharmakeerthi, C.H.; Mithulananthan, N.; Saha, T.K. Impact of electric vehicle fast charging on power system voltage stability. Int. J. Electr. Power Energy Syst. 2014, 57, 241-249. [CrossRef]

6. Liu, N.; Chen, Q.; Liu, J.; Lu, X.Y.; Li, P.; Lei, J.Y.; Zhang, J.H. A heuristic operation strategy for commercial building microgrids containing EVs and PV system. IEEE Trans. Ind. Electron. 2015, 62, 2560-2570. [CrossRef]

7. Nguyen, H.K.; Song, J.B. Optimal charging and discharging for multiple PHEVs with demand side management in vehicle-to-building. J. Commun. Netw. 2012, 14, 662-671. [CrossRef]

8. Han, S.; Han, S.; Sezaki, K. Development of an optimal vehicle-to-grid aggregator for frequency regulation. IEEE Trans. Smart Grid 2010, 1, 65-72.

9. Clement-Nyns, K.; Haesen, E.; Driesen, J. The impact of charging plug-in hybrid electric vehicles on a residential distribution grid. IEEE Trans. Power Syst. 2010, 25, 371-380. [CrossRef]

10. Deilami, S.; Masoum, A.S.; Moses, P.S.; Masoum, M.A.S. Realtime coordination of plug-in electric vehicle charging in smart grids to minimize power losses and improve voltage profile. IEEE Trans. Smart Grid 2011, 2, 456-467. [CrossRef]

11. Ota, Y.; Taniguchi, H.; Nakajima, T.; Liyanage, K.; Baba, J.; Yokoyama, A. Autonomous distributed v2g (vehicle-to-grid) consideringn charging request and battery condition. In Proceedings of the 2010 IEEE PES Innovative Smart Grid Technologies Conference Europe (ISGT Europe), Gothenburg, Sweden, 11 October 2010; pp. 1-6.

12. Singh, M.; Kumar, P.; Kar, I. Implementation of vehicle to grid infrastructure using fuzzy logic controller. IEEE Trans. Smart Grid 2012, 3, 565-577. [CrossRef]

13. Wu, C.; Mohsenian-Rad, H.; Huang, J. Vehicle-to-aggregator interaction game. IEEE Trans. Smart Grid 2012, 3, 434-442. [CrossRef]

14. Qi, X.; Wu, G.; Boriboonsomsin, K.; Barth, M.J. Development and Evaluation of an Evolutionary Algorithm-Based OnLine Energy Management System for Plug-In Hybrid Electric Vehicles. IEEE Trans. Intell. Transp. Syst. 2017, 18, 2181-2191. [CrossRef]

15. Wang, X.; Liang, Q. Energy management strategy for plug-in hybrid electric vehicles via bidirectional vehicle-to-grid. IEEE Syst. J. 2017, 11, 1789-1798. [CrossRef]

16. Badawy, M.O. Power Flow Management of a Grid Tied PV-Battery Powered Fast Electric Vehicle Charging Station. IEEE Trans. Ind. Appl. 2017, 53, 4959-4966. [CrossRef]

17. Shojaabadi, S.; Abapour, S.; Abapour, M.; Nahavandi, A. Simultaneous planning of plug-in hybrid electric vehicle charging stations and wind power generation in distribution networks considering uncertainties. Renew. Energy 2016, 99, 237-252. [CrossRef]

18. Rahbari-Asr, N.; Chen, J.; Deng, R. Distributed real-time pricing control for large-scale unidirectional V2G with multiple energy suppliers. IEEE Trans. Ind. Inform. 2016, 12, 1953-1962. [CrossRef]

19. Quilumba, F.L.; Lee, W.J.; Huang, H.; Wang, D.Y.; Szabados, R.L. Using Smart Meter Data to Improve the Accuracy of Intraday Load Forecasting Considering Customer Behavior Similarities. IEEE Trans. Smart Grid 2015, 6, 911-918. [CrossRef]

20. Su, W.; Chow, M.Y. Computational intelligence-based energy management for a large-scale PHEV/PEV enabled municipal parking deck. Appl. Energy 2012, 96, 171-182. [CrossRef]

21. Jiménez-Bermejo, D.; Fraile-Ardanuy, J.; Castaño-Solis, S.; Merino, J.; Álvaro-Hermana, R. Using Dynamic Neural Networks for Battery State of Charge Estimation in Electric Vehicles. Procedia Comput. Sci. 2018, 130, 533-540. [CrossRef]

22. Jahangir, H.; Tayarani, H.; Ahmadian, A.; Golkar, M.A.; Miret, J.; Tayarani, M.; Gao, H.O. Charging demand of Plug-in Electric Vehicles: Forecasting travel behavior based on a novel Rough Artificial Neural Network approach. J. Clean. Prod. 2019, 229, 1029-1044. [CrossRef]

23. Zhao, J.X.; Ma, Y.Y.; Zhang, Z.D.; Wang, S.W.; Wang, S. Optimization and matching for range-extenders of electric vehicles with artificial neural network and genetic algorithm. Energy Convers. Manag. 2019, 184, 709-725. [CrossRef]

(C) 2019 by the authors. Licensee MDPI, Basel, Switzerland. This article is an open access article distributed under the terms and conditions of the Creative Commons Attribution (CC BY) license (http://creativecommons.org/licenses/by/4.0/). 